\title{
NEW BOUNDS ON THE LIEB-THIRRING CONSTANTS
}

\author{
D. HUNDERTMARK ${ }^{1}$, A. LAPTEV ${ }^{2}$ AND T. WEIDL ${ }^{2,3}$
}

\begin{abstract}
Improved estimates on the constants $\mathrm{L}_{\gamma, \mathrm{d}}$, for $1 / 2<$ $\gamma<3 / 2, d \in N$ in the inequalities for the eigenvalue moments of Schrödinger operators are established.
\end{abstract}

\section{INTRODUCTION}

Let us consider a Schrödinger operator in $\mathrm{L}^{2}\left(\mathbb{R}^{\mathrm{d}}\right)$

$$
-\Delta+\mathrm{V},
$$

where $\mathrm{V}$ is a real-valued function. The inequalities

$$
\operatorname{tr}(-\Delta+\mathrm{V})_{-}^{\gamma} \leq \mathrm{L}_{\gamma, \mathrm{d}} \int_{\mathbb{R}^{\mathrm{d}}} \mathrm{V}_{-}^{\gamma+\frac{\mathrm{d}}{2}} \mathrm{~d} x,
$$

are known as Lieb-Thirring bounds and hold true with finite constants $\mathrm{L}_{\gamma, \mathrm{d}}$ if and only if $\gamma \geq 1 / 2$ for $d=1, \gamma>0$ for $d=2$ and $\gamma \geq 0$ for $d \geq 3$. Here and in the following, $A_{ \pm}=(|A| \pm A) / 2$ denote the positive and negative parts of a self-adjoint operator $A$. The case $\gamma>(1-d / 2)_{+}$ was shown by Lieb and Thirring in [21]. The critical case $\gamma=0, d \geq 3$ is known as the Cwikel-Lieb-Rozenblum inequality, see [8, 19, 22] and also [18, 7]. The remaining case $\gamma=1 / 2, d=1$ was verified in [25].

It is known that as soon as $V \in \mathrm{L}^{\gamma+\mathrm{d} / 2}\left(\mathbb{R}^{\mathrm{d}}\right)$ and the constant $\mathrm{L}_{\gamma, \mathrm{d}}$ is finite, then we have Weyl's asymptotic formula

$$
\begin{aligned}
\lim _{\alpha \rightarrow+\infty} \frac{1}{\alpha^{\gamma+\frac{d}{2}}} \operatorname{tr}(-\Delta+\alpha V)_{-}^{\gamma} & =\lim _{\alpha \rightarrow+\infty} \frac{1}{\alpha^{\gamma+\frac{d}{2}}} \iint_{\mathbb{R}^{\mathrm{d}} \times \mathbb{R}^{\mathrm{d}}}\left(|\xi|^{2}+\alpha \mathrm{V}\right)_{-}^{\gamma} \frac{\mathrm{d} x \mathrm{~d} \xi}{(2 \pi)^{\mathrm{d}}} \\
& =\mathrm{L}_{\gamma, \mathrm{d}}^{\mathrm{cl}} \int_{\mathbb{R}^{\mathrm{d}}} \mathrm{V}_{-}^{\gamma+\frac{\mathrm{d}}{2}} \mathrm{dx},
\end{aligned}
$$

where the so-called classical constant $L_{\gamma, \mathrm{d}}^{\mathrm{cl}}$ is defined by

$$
\mathrm{L}_{\gamma, \mathrm{d}}^{\mathrm{cl}}=(2 \pi)^{-\mathrm{d}} \int_{\mathbb{R}^{\mathrm{d}}}\left(|\xi|^{2}-1\right)_{-}^{\gamma} \mathrm{d} \xi=\frac{\Gamma(\gamma+1)}{2^{\mathrm{d}} \pi^{\mathrm{d} / 2} \Gamma\left(\gamma+\frac{\mathrm{d}}{2}+1\right)}, \quad \gamma \geq 0 .
$$

This immediately implies $\mathrm{L}_{\gamma, \mathrm{d}}^{\mathrm{cl}} \leq \mathrm{L}_{\gamma, \mathrm{d}}$.

1991 Mathematics Subject Classification. Primary 35P15; Secondary 35L15, 47A75, $35 \mathrm{~J} 10$. 
Until recently the sharp values of $\mathrm{L}_{\gamma, \mathrm{d}}$ were known only for $\gamma \geq 3 / 2$, $\mathrm{d}=1$, (see [21, 1]), where they coincide with $\mathrm{L}_{\gamma, \mathrm{d}}^{\mathrm{cl}}$. In [17] Laptev and Weidl extended this result to all dimensions. They proved that $\mathrm{L}_{\gamma, \mathrm{d}}=\mathrm{L}_{\gamma, \mathrm{d}}^{\mathrm{cl}}$, for $\gamma \geq 3 / 2, d \in \mathbb{N}$. Recently, Hundertmark, Lieb and Thomas showed in [15] that the sharp value of $L_{1 / 2,1}$ is equal to $1 / 2$.

The purpose of this paper is to give some new bounds on the constants $\mathrm{L}_{\gamma, \mathrm{d}}$ for $1 / 2<\gamma<3 / 2$ and all $\mathrm{d} \in \mathbb{N}$ (see $\S 4$ ). In particular, one of our main results given in Theorem 4.1, says that

$$
\mathrm{L}_{\gamma, \mathrm{d}} \leq 2 \mathrm{~L}_{\gamma, \mathrm{d}}^{\mathrm{cl}}, \quad 1 \leq \gamma<3 / 2, \quad \mathrm{~d} \in \mathbb{N},
$$

whereas for large dimensions it was only known that $L_{\gamma, d} \leq C \sqrt{d} L_{\gamma, d}^{c l}$ with some constant $\mathrm{C}>0$.

For the important case $\gamma=1, \mathrm{~d}=3$ we have $\mathrm{L}_{1,3} \leq 2 \mathrm{~L}_{1,3}^{\mathrm{cl}}<0.013509$ compared with $\mathrm{L}_{1,3}<5.96677 \mathrm{~L}_{1,3}^{\mathrm{cl}}<0.040303$ obtained in [20] and its improvement $\mathrm{L}_{1,3}<5.21803 \mathrm{~L}_{1,3}^{\mathrm{cl}}<0.035246$ obtained in [5].

Note also that our estimates on the constant $\mathrm{L}_{\gamma, \mathrm{d}}$ imply that $\mathrm{L}_{1, \mathrm{~d}} \leq$ $2 \mathrm{~L}_{1, \mathrm{~d}}^{\mathrm{cl}}<\mathrm{L}_{0, \mathrm{~d}}^{\mathrm{cl}}$ as was conjectured in [23].

In order to obtain our results we give a version of the proof obtained in [15] for matrix-valued potentials (see $\S 3$ ). Note that E.H.Lieb has informed us that the original proof obtained in [15] also works for matrix-valued potentials. After that in $\S 4$ we apply the equality $\mathrm{L}_{0, \mathrm{~d}}=\mathrm{L}_{0, \mathrm{~d}}^{\mathrm{cl}}$, for $\gamma \geq 3 / 2$ and $d \in \mathbb{N}$ shown in [17] by using the "lifting" argument with respect to the dimension d suggested in [16]. The same arguments as in [17] yield the corresponding inequalities for Schrödinger operators with magnetic fields.

In $\S 5$ we recover the matrix-valued version of the Buslaev-FaddeevZakharov trace formulae obtained in [17] and find some new two sides spectral inequalities for one-dimensional Schrödinger operators with operatorvalued potentials.

Finally, we are very grateful to L.E.Thomas who was also involved in the new proof of Theorem 3.1 and has written $\S 3.4$ as well as reading the text of the paper and making many valuable remarks.

\section{NotATION AND AUXILIARY MATERiAL}

Let $\mathbf{G}$ be a separable Hilbert space with the norm $\|\cdot\|_{\mathbf{G}}$ and the scalar product $\langle\cdot, \cdot\rangle_{\mathbf{G}}$ and let $\mathbf{O}_{\mathbf{G}}$ and $\mathbf{1}_{\mathbf{G}}$ be the zero and the identity operator on $\mathbf{G}$. Denote by $\mathcal{B}(\mathbf{G})$ the Banach space of all bounded operators on $\mathbf{G}$ and by $\mathcal{K}(\mathbf{G})$ the (separable) ideal of all compact operators. Let $\mathcal{S}_{1}(\mathbf{G})$ and $\mathcal{S}_{2}(\mathbf{G})$ be the classes of trace and Hilbert-Schmidt operators on $\mathbf{G}$ respectively. For a nonnegative operator $A \in \mathcal{K}(\mathbf{G})$

$$
\lambda_{1}(A) \geq \lambda_{2}(A) \geq \ldots \geq 0
$$


is the ordered sequence of its eigenvalues (including multiplicities). We use the symbol "tr" to denote traces of operators (matrices) in different Hilbert spaces.

The Hilbert space $\mathbf{H}=\mathrm{L}^{2}\left(\mathbb{R}^{\mathrm{d}}, \mathbf{G}\right)$ is the space of all measurable functions $u: \mathbb{R}^{\mathrm{d}} \rightarrow \mathbf{G}$ such that

$$
\|\mathrm{u}\|_{\mathbf{H}}^{2}:=\int_{\mathbb{R}^{\mathrm{d}}}\|\mathrm{u}\|_{\mathbf{G}}^{2} \mathrm{~d} x<\infty .
$$

The Sobolev space $H^{1}\left(\mathbb{R}^{d}, \mathbf{G}\right)$ consists of all functions $u \in H$ whose norm

$$
\|u\|_{H^{1}\left(\mathbb{R}^{d}, \mathbf{G}\right)}^{2}=\sum_{k=1}^{d}\left\|\partial u / \partial x_{k}\right\|_{H^{2}}^{2}+\|u\|_{H^{2}}^{2}
$$

is finite. Obviously the quadratic form

$$
h[u, u]=\sum_{k=1}^{d}\left\|\partial u / \partial x_{k}\right\|_{\mathbf{H}}^{2}
$$

is closed in $L^{2}\left(\mathbb{R}^{d}, \mathbf{G}\right)$ on the domain $u \in H^{1}\left(\mathbb{R}^{d}, \mathbf{G}\right)$. Let

$$
\mathrm{V}(\cdot): \mathbb{R}^{\mathrm{d}} \rightarrow \mathrm{B}(\mathbf{G})
$$

be an operator-valued function satisfying

$$
\|\mathrm{V}(\cdot)\|_{\mathrm{B}(\mathbf{G})} \in \mathrm{L}^{\mathrm{p}}\left(\mathbb{R}^{\mathrm{d}}\right)
$$

for some finite $p$ with

$$
\begin{aligned}
& p \geq 1 \text { if } d=1 \\
& p>1 \text { if } d=2 \\
& p \geq d / 2 \text { if } d \geq 3
\end{aligned}
$$

Then the quadratic form

$$
v[u, u]=\int_{\mathbb{R}^{\mathrm{d}}}\langle\mathrm{Vu}, \mathrm{u}\rangle_{\mathbf{G}} \mathrm{dx}
$$

is bounded with respect to $h[\cdot, \cdot]$ and thus the form

$$
h[u, u]+v[u, u]
$$

is closed and semi-bounded from below on $H^{1}\left(\mathbb{R}^{\mathrm{d}}, \mathbf{G}\right)$. It generates the self-adjoint operator

$$
\mathrm{Q}=-\left(\Delta \otimes \mathbf{1}_{\mathbf{G}}\right)+\mathrm{V}(\mathrm{x})
$$

in $L^{2}\left(\mathbb{R}^{d}, \mathbf{G}\right)$. It is not difficult to see, that if the operator $V(x)$ belongs to $\mathcal{K}(\mathbf{G})$ for a.e. $x \in \mathbb{R}^{\mathrm{d}}$ and satisfies the condition (2.1), then the negative spectrum

$$
-\mathrm{E}_{1} \leq-\mathrm{E}_{2} \leq \cdots \leq-\mathrm{E}_{\mathrm{n}} \leq \cdots<0
$$


of the operator $\mathrm{Q}$ is discrete.

3. AN UPPER BOUND FOR THE EIGENVALUE MOMENT IN THE CRITICAL CASE $d=1$ AND $\gamma=1 / 2$.

3.1. A sharp Lieb-Thirring inequality for $d=1$ and $\gamma=1 / 2$. In this section we give a version of the proof from [15]] which will be applied to the Schrödinger operators with operator-valued potentials. The main result of this section is the following statement:

Theorem 3.1. Let $\mathrm{V}(\mathrm{x})$ be a nonpositive operator-valued function, such that $\mathrm{V}(\mathrm{x}) \in \mathcal{S}_{1}(\mathbf{G})$ for a.e. $\mathrm{x} \in \mathbb{R}$ and $\operatorname{tr} \mathrm{V}_{-}(\cdot) \in \mathrm{L}^{1}(\mathbb{R})$. Then

$$
\operatorname{tr}\left(-\frac{\mathrm{d}^{2}}{\mathrm{~d} x^{2}} \otimes 1_{G}+V\right)_{-}^{1 / 2}=\sum_{j} \sqrt{E_{j}} \leq \frac{1}{2} \int_{-\infty}^{\infty} \operatorname{tr} V_{-} d x
$$

Remark. The constant $\mathrm{L}_{1 / 2,1}=1 / 2=2 \mathrm{~L}_{1 / 2,1}^{\mathrm{cl}}$ is the best possible. Indeed, $1 / 2$ is achieved by the operator of rank one $\mathrm{V}(x)=\delta(x)\langle\cdot, e\rangle e$, where $e \in \mathbf{G}$ and $\delta$ is Dirac's $\delta$-function (see [15]).

We follow the strategy of [15] quite closely but give a different proof of the monotonicity lemma.

3.2. Monotonicity Lemma. In order to prove the monotonicity lemma we need an auxiliary "majorization" result. Let $A \in \mathcal{K}(\mathbf{G})$ and let us denote

$$
\|A\|_{n}=\sum_{j=1}^{n} \sqrt{\lambda_{j}\left(A^{*} A\right)}
$$

Then by Ky-Fan's inequality (see for example [112, Lemma 4.2]) the functionals $\|\cdot\|_{n}, n=1,2, \ldots$, are norms on $\mathcal{K}(\mathbf{G})$ and thus for any unitary operator $\mathbf{U}$ in $\mathbf{G}$ we have

$$
\left\|\mathrm{U}^{*} \mathrm{AU}\right\|_{n}=\|\mathrm{A}\|_{n}
$$

Definition 3.2. Let $A, B$ be two compact operators on $\mathbf{G}$. We say that $A$ majorizes $\mathrm{B}$ or $\mathrm{B} \prec \mathrm{A}$, iff

$$
\|\mathrm{B}\|_{\mathrm{n}} \leq\|\mathrm{A}\|_{\mathrm{n}} \quad \text { for all } \mathrm{n} \in \mathbb{N} \text {. }
$$

Lemma 3.3 (Majorization). Let A be a nonnegative compact operator $\mathbf{G}$, $\{\mathrm{U}(\omega)\}_{\omega \in \Omega}$ be a family of unitary operators on $\mathbf{G}$, and let $\mathrm{g}$ be a probability measure on $\Omega$. Then the operator

$$
\mathrm{B}:=\int_{\Omega} \mathrm{U}^{*}(\omega) \operatorname{AU}(\omega) \mathrm{g}(\mathrm{d} \omega)
$$

is majorized by $\mathrm{A}$. 
Proof. This is a simple consequence of the triangle inequality

$$
\|\mathrm{B}\|_{n} \leq \int_{\Omega}\left\|\mathrm{U}^{*}(\omega) A \mathrm{U}(\omega)\right\|_{n} \mathrm{~g}(\mathrm{~d} \omega)=\mathrm{g}(\Omega)\|A\|_{n}=\|A\|_{n} .
$$

Remark. The notion of majorization is well-known in matrix theory (see [3]). For finite dimensional Hilbert spaces $\mathbf{G}$ even the converse statement of Lemma 3.3 is true, cf. [2, Theorem 7.1]:

If $A$ and $B$ are nonnegative matrices and $\operatorname{tr} A=\operatorname{tr} B$, then the condition $B \prec A$ implies that there exist unitary matrices $U_{j}$ and $t_{j}>0, j=1, \ldots, N$, such that

$$
\sum_{j=1}^{N} t_{j}=1, \quad B=\sum_{j=1}^{N} t_{j} u_{j}^{*} A u_{j} .
$$

Let $W(\cdot): \mathbb{R} \rightarrow \mathcal{S}_{2}(\mathbf{G})$ be an operator-valued function and let $\|\mathrm{W}(\cdot)\|_{\mathcal{S}_{2}} \in \mathrm{L}^{2}(\mathbb{R})$. Denote

$$
\mathcal{L}_{\varepsilon}:=W^{*}\left[2 \varepsilon\left(-\frac{\mathrm{d}^{2}}{\mathrm{~d} x^{2}}+\varepsilon^{2}\right)^{-1} \otimes 1_{\mathbf{G}}\right] W .
$$

Obviously, $\mathcal{L}_{\varepsilon}$ is a nonnegative, trace class operator on $\mathrm{L}^{2}(\mathbb{R}, \mathbf{G})$, its trace is independent of $\varepsilon, 0 \leq \varepsilon<\infty$ and equals $\operatorname{tr} \mathcal{L}_{\varepsilon}=\int\|W(x)\|_{\mathcal{S}_{2}}^{2} \mathrm{~d} x$.

Lemma 3.4 (Monotonicity). The operator $\mathcal{L}_{\varepsilon}$ is majorized by $\mathcal{L}_{\varepsilon^{\prime}}$

$$
\mathcal{L}_{\varepsilon} \prec \mathcal{L}_{\varepsilon^{\prime}}
$$

for all $0 \leq \varepsilon^{\prime} \leq \varepsilon$.

Proof. Using the majorization Lemma 3.3 the proof is basically reduced to a right choice of notation. Let $A$ be the nonnegative compact operator in $L^{2}(\mathbb{R}, \mathbf{G})$, given by the integral kernel[ $A(x, y):=W^{*}(x) W(y)$. Furthermore let

$$
g_{\varepsilon}(d p)=\left\{\begin{array}{lll}
\varepsilon\left(\pi\left(p^{2}+\varepsilon^{2}\right)\right)^{-1} d p & \text { if } & \varepsilon>0 \\
\delta(d p) & \text { if } & \varepsilon=0
\end{array}\right.
$$

be the Cauchy distribution and $\{\mathrm{U}(\mathrm{p})\}_{\mathrm{p} \in \mathbb{R}}$ be the group of unitary multiplication operators $(U(p) \psi)(x)=e^{-i p x} \psi(x)$ on $L^{2}(\mathbb{R}, \mathbf{G})$. Passing to the Fourier representation of the Green function in (3.2) we obtain

$$
\mathcal{L}_{\varepsilon}=\int_{-\infty}^{\infty} U^{*}(p) A u(p) g_{\varepsilon}(d p) .
$$

\footnotetext{
${ }^{1}$ In the scalar case $A$ would just be the rank one operator $|W\rangle\langle W|$ (in Dirac notation).
} 
Of course, $\mathcal{L}_{0}=A$. In particular, Lemma 3.3 and (3.4) immediately imply $\mathcal{L}_{\varepsilon} \prec \mathcal{L}_{0}$. The Cauchy distribution is a convolution semigroup, i.e. $g_{\varepsilon}=$ $g_{\varepsilon}{ }^{\prime} * g_{\varepsilon-\varepsilon^{\prime}}$. If we insert this into (3.4) and change variables using the group property of the unitary operators $\mathrm{U}(\mathrm{p})$, then Lemma 3.3 yields

$$
\mathcal{L}_{\varepsilon}=\int \mathrm{U}^{*}(p) \mathcal{L}_{\varepsilon^{\prime}} \mathrm{U}(\mathrm{p}) \mathrm{g}_{\varepsilon-\varepsilon^{\prime}}(\mathrm{p}) \mathrm{d} p \prec \mathcal{L}_{\varepsilon^{\prime}}
$$

This completes the proof.

3.3. Proof of Theorem 3.1. Let $W(x)=\sqrt{V_{-}(x)}$, so $W^{*}=W$. Then from the assumptions made in Theorem 3.1, we find that $W(x)$ is a family of nonnegative Hilbert-Schmidt operators such that $\|W(\cdot)\|_{\mathcal{S}_{2}} \in \mathrm{L}^{2}(\mathbb{R})$. Let

$$
\mathcal{K}_{\mathrm{E}}:=\frac{1}{2 \sqrt{\mathrm{E}}} \mathcal{L}_{\sqrt{\mathrm{E}}}=\mathrm{W}\left[\left(-\frac{\mathrm{d}^{2}}{\mathrm{dx^{2 }}}+\mathrm{E}\right)^{-1} \otimes \mathbf{1}_{\mathrm{G}}\right] \mathrm{W},
$$

where $\mathcal{L}_{\varepsilon}$ is defined in (3.2). According to the Birman-Schwinger principle [曰, 24] we have

$$
1=\lambda_{j}\left(\mathcal{K}_{E_{j}}\right)
$$

for all negative eigenvalues $\left\{-E_{j}\right\}_{j}$ of the Schrödinger operator (2.3). Multiplying this equality by $2 \sqrt{E_{j}}$ and summing over $j$ we obtain

$$
2 \sum \sqrt{E_{j}}=\sum \lambda_{j}\left(\mathcal{L}_{\sqrt{E_{j}}}\right)
$$

In contrast to $\mathcal{K}_{\mathrm{E}}$ the operator $\mathcal{L}_{\sqrt{E}}$ is well-behaved for small energies. We now use the same monotonicity argument as in [15] to dispose of the energy dependence of the operator in (3.6). Namely, for any $n \in \mathbb{N}$, Lemma 3.4 implies that the partial traces $\sum_{j \leq n} \lambda_{j}\left(\mathcal{L}_{\varepsilon}\right)$ are monotone decreasing in $\varepsilon$. Given this monotonicity, a simple induction argument yields

$$
\sum_{j \leq n} \lambda_{j}\left(\mathcal{L}_{\sqrt{E_{j}}}\right) \leq \sum_{j \leq n} \lambda_{j}\left(\mathcal{L}_{\sqrt{E_{n}}}\right) \quad \text { for all } n \in \mathbb{N} .
$$

Hence, by (3.6) we also have the bound

$$
2 \sum \sqrt{E_{j}} \leq \sum \lambda_{j}\left(\mathcal{L}_{0}\right)=\operatorname{tr} \mathcal{L}_{0}=\int_{-\infty}^{\infty} \operatorname{tr} W^{2}(x) d x=\int_{-\infty}^{\infty} \operatorname{tr} V_{-}(x) d x
$$

The proof is complete.

3.4. Some generalizations of Theorem 3.1. The above strategy can be adapted to obtain upper bounds on eigenvalue moments for operators of the form $H=|-i \nabla|^{\beta}+V$ acting in $L^{2}\left(R^{d}\right), \beta>d$. Suppose that $\Phi$ is an infinitely divisible symmetric probability density, e.g. a compound Poisson, of the form

$$
\Phi(p)=\frac{1}{(2 \pi)^{d}} \int e^{\int(\cos (x \cdot \xi)-1) d m(\xi)} e^{i p \cdot x} d x
$$


with $m$ a non-negative measure and such that $\Phi$ satisfies a point-wise inequality

$$
\left(|p|^{\beta}+1\right)^{-1} \leq c_{0} \Phi(p)
$$

for some constant $\mathrm{c}_{0}$. Then by scaling,

$$
E^{\frac{\beta-d}{\beta}}\left(|p|^{\beta}+E\right)^{-1} \leq c_{0} E^{-d / \beta} \Phi\left(p / E^{\beta}\right) \equiv \Psi_{E}(p)
$$

Moreover,

$$
\Psi_{E}(p) \equiv \Theta_{E, E^{\prime}} * \Psi_{E^{\prime}}(p)
$$

where $\Theta_{E, E^{\prime}}$ is a non-negative probability density with Fourier transform given by

$$
\widehat{\Theta}_{\mathrm{E}, \mathrm{E}^{\prime}}(x)=\exp \left\{\int(\cos (x \cdot \xi)-1)\left[\mathrm{dm}\left(\xi / \mathrm{E}^{1 / \beta}\right)-\mathrm{dm}\left(\xi / \mathrm{E}^{\prime 1 / \beta}\right)\right]\right\}
$$

provided that $\left[\mathrm{dm}\left(\xi / E^{1 / \beta}\right)-\mathrm{dm}\left(\xi / E^{\prime 1 / \beta}\right)\right]$ is non-negative for $E^{\prime} \leq E$.

Assuming that $\mathrm{dm}$ satisfies this condition, we have by the majorization argument that

$$
\begin{aligned}
\sum_{j \leq n} E_{j}^{(\beta-d) / \beta}(H) & \leq \sum_{j \leq n} \lambda_{j}\left(V_{-}^{1 / 2} \frac{E_{j}^{(\beta-d) / \beta}}{|-i \nabla|^{\beta}+E_{j}} V_{-}^{1 / 2}\right) \\
& \leq \sum_{j \leq n} \lambda_{j}\left(V_{-}^{1 / 2} \Psi_{E_{j}}(-i \nabla) V_{-}^{1 / 2}\right) \\
& \leq \operatorname{tr}\left(V_{-}^{1 / 2} \Psi_{E_{n}}(-i \nabla) V_{-}^{1 / 2}\right)=\frac{c_{0}}{(2 \pi)^{d}} \int V_{-}(x) d x
\end{aligned}
$$

The problem of finding such an optimal $\Phi$ and $c_{0}$ seems non-trivial in general. But in $d$ dimensions, with the choice $\mathrm{dm}(\xi)=\mathrm{cd} \xi /|\xi|^{\mathrm{d}+\alpha}$, with $\mathrm{d}+\alpha \leq \beta, 0<\alpha<2,(\cos (x \cdot \xi)-1)$ is integrable with respect to $\mathrm{m}$, and $\int(\cos (x \cdot \xi)-1) d m(\xi)=-c_{1}|x|^{\alpha}$ for some $c_{1}>0$. Consequently, $\Phi(p) \sim$ $|p|^{-(\alpha+d)}, p \rightarrow \infty$, and $c_{0} \Phi$ will majorize $\left(|p|^{\beta}+1\right)^{-1}$ for sufficiently large $c_{0}$. An eigenvalue moment bound (3.8) follows. For the $d=1, \beta=2$ Cauchy density case above, the optimal choice is $\mathrm{dm}(\xi)=\mathrm{d} \xi /\left(\pi \xi^{2}\right)$ and $\mathrm{c}_{0}=\pi ;(3.7)$ is an equality.

3.5. A priori estimate for moments $\gamma \geq \mathbf{1} / \mathbf{2}$. Following Aizenman and Lieb [1] we can "lift" the bound of Theorem 3.1 to moments $\gamma \geq 1 / 2$. 
Corollary 3.5. Assume that $\mathrm{V}(\mathrm{x})$ is a nonpositive operator-valued function for a.e. $x \in \mathbb{R}$ and that $\operatorname{tr} \mathrm{V}_{-}(\cdot) \in \mathrm{L}^{\gamma+\frac{1}{2}}(\mathbb{R})$ for some $\gamma \geq 1 / 2$. Then

$$
\operatorname{tr}\left(-\frac{\mathrm{d}^{2}}{\mathrm{~d} x^{2}} \otimes \mathbf{1}_{\mathrm{G}}+\mathrm{V}\right)_{-}^{\gamma}=\sum_{j} \mathrm{E}_{j}^{\gamma} \leq 2 \mathrm{~L}_{\gamma, 1}^{\mathrm{cl}} \int_{-\infty}^{\infty} \operatorname{tr} \mathrm{V}_{-}^{\gamma+\frac{1}{2}} \mathrm{dx} .
$$

Proof. Note that Theorem 3.1 is equivalent to

$$
\operatorname{tr}\left(-\frac{\mathrm{d}^{2}}{\mathrm{~d} x^{2}} \otimes 1_{\mathrm{G}}+\mathrm{V}\right)_{-}^{1 / 2} \leq 2 \iint_{\mathbb{R} \times \mathbb{R}} \operatorname{tr}\left(\mathrm{p}^{2}-\mathrm{V}_{-}(\mathrm{x})\right)_{-}^{1 / 2} \frac{\mathrm{dpd} x}{2 \pi}
$$

Scaling gives the simple identity for all $s \in \mathbb{R}$

$$
s_{-}^{\gamma}=C_{\gamma} \int_{0}^{\infty} t^{\gamma-\frac{3}{2}}(s+t)_{-}^{1 / 2} d t, \quad C_{\gamma}^{-1}=B\left(\gamma-\frac{1}{2}, \frac{3}{2}\right),
$$

where $B$ is the Beta function. Let $\mu_{j}(x)$ the eigenvalues of $V_{-}(x)$. Then

$$
\begin{aligned}
& \operatorname{tr}\left(-\frac{d^{2}}{d x^{2}} \otimes 1_{G}+V\right)_{-}^{\gamma}=C_{\gamma} \int_{0}^{\infty} d t t^{\gamma-\frac{3}{2}} \operatorname{tr}\left(-\frac{d^{2}}{d x^{2}} \otimes 1_{G}+V+t\right)_{-}^{1 / 2} \\
& \leq C_{\gamma} \int_{0}^{\infty} d t t^{\gamma-\frac{3}{2}} 2 \iint \operatorname{tr}\left(p^{2}-V_{-}+t\right)_{-}^{1 / 2} \frac{d p d x}{2 \pi} \\
& =2 \sum_{j=1}^{\infty} \iint\left[C_{\gamma} \int_{0}^{\infty} d t t^{\gamma-\frac{3}{2}}\left(p^{2}-\mu_{j}+t\right)_{-}^{1 / 2}\right] \frac{d p d x}{2 \pi} \\
& =2 \iint \operatorname{tr}\left(\mathrm{p}^{2}-\mathrm{V}_{-}\right)_{-}^{\gamma} \frac{\mathrm{dpdx}}{2 \pi}=2 \mathrm{~L}_{\gamma, 1}^{\mathrm{cl}} \int \operatorname{tr} \mathrm{V}_{-}^{\gamma+1 / 2} \mathrm{dx} \text {. }
\end{aligned}
$$

4. New estimates On the CONSTANTS $\mathrm{L}_{\gamma, \mathrm{d}}$ FOR $1 / 2 \leq \gamma<3 / 2$,

$$
\mathrm{d} \in \mathbb{N}
$$

4.1. The Main result. We consider now the Schrödinger operator (2.3) in $\mathrm{L}^{2}\left(\mathbb{R}^{\mathrm{d}}, \mathbf{G}\right)$ for an arbitrary $\mathrm{d} \in \mathbb{N}$. Assume that $\mathrm{V}$ is a nonpositive operatorvalued function satisfying the condition

$$
\operatorname{tr} \mathrm{V}(\cdot) \in \mathrm{L}^{\gamma+\frac{\mathrm{d}}{2}}\left(\mathbb{R}^{\mathrm{d}}\right)
$$

for some appropriate $\gamma$. We shall discuss bounds on the optimal constants in the Lieb-Thirring inequalities

$$
\operatorname{tr}(-\Delta \otimes 1+V)_{-}^{\gamma} \leq \mathrm{L}_{\gamma, \mathrm{d}} \int_{\mathbb{R}^{\mathrm{d}}} \operatorname{tr} \mathrm{V}_{-}^{\frac{\mathrm{d}}{2}+\gamma} \mathrm{dx}
$$

In [17] it has been shown that

$$
\mathrm{L}_{\gamma, \mathrm{d}}=\mathrm{L}_{\gamma, \mathrm{d}}^{\mathrm{cl}} \quad \text { for all } \quad \gamma \geq 3 / 2, \quad \mathrm{~d} \in \mathbb{N} .
$$

The main result of the paper concerns $1 / 2 \leq \gamma<3 / 2$. 
Theorem 4.1. Let $\mathrm{V}$ be a nonpositive operator-valued function and let the condition (4.1) be satisfied. Then the following estimates on the sharp constants $\mathrm{L}_{\gamma, \mathrm{d}}$ hold

$$
\begin{array}{lrrr}
\mathrm{L}_{\gamma, \mathrm{d}} \leq 2 \mathrm{~L}_{\gamma, \mathrm{d}}^{\mathrm{cl}} & \text { for all } & 1 \leq \gamma<3 / 2, & \mathrm{~d} \in \mathbb{N}, \\
\mathrm{L}_{\gamma, \mathrm{d}} \leq 2 \mathrm{~L}_{\gamma, \mathrm{d}}^{\mathrm{cl}} & \text { for all } & 1 / 2 \leq \gamma<3 / 2, & \mathrm{~d}=1, \\
\mathrm{~L}_{\gamma, \mathrm{d}} \leq 4 \mathrm{~L}_{\gamma, \mathrm{d}}^{\mathrm{cl}} & \text { for all } & 1 / 2 \leq \gamma<1, & \mathrm{~d} \geq 2 .
\end{array}
$$

Remark. For the special case $\gamma=1$ we find that

$$
\mathrm{L}_{1, \mathrm{~d}}^{\mathrm{cl}} \leq \mathrm{L}_{1, \mathrm{~d}} \leq 2 \mathrm{~L}_{1, \mathrm{~d}}^{\mathrm{cl}} \quad \text { for all } \mathrm{d} \in \mathbb{N} \text {. }
$$

Even in the scalar case $\mathbf{G}=\mathbb{C}$ this is a substantial improvement of the previously known numerical estimates on these constants in high dimensions obtained in [5] and [20].

Remark. In fact, our proof of Theorem 4.1 yields

$$
\mathrm{L}_{\gamma, \mathrm{d}} \leq \frac{\mathrm{L}_{\gamma, 1}}{\mathrm{~L}_{\gamma, 1}^{\mathrm{cl}}} \mathrm{L}_{\gamma, \mathrm{d}}^{\mathrm{cl}}, \quad \mathrm{d} \in \mathbb{N}, \quad 1 \leq \gamma<3 / 2 .
$$

According to Corollary 3.5 we know that $\mathrm{L}_{1,1} \leq 2 \mathrm{~L}_{1,1}^{\mathrm{cl}}$. In the scalar case Lieb and Thirring conjectured that

$$
\frac{\mathrm{L}_{\gamma, 1}}{\mathrm{~L}_{\gamma, 1}^{\mathrm{cl}}}=2\left(\frac{\gamma-1 / 2}{\gamma+1 / 2}\right)^{\gamma-1 / 2}, \quad 1 / 2 \leq \gamma<3 / 2 .
$$

In particular, if this were true in the matrix case for $\gamma=1$, our approach would imply $\mathrm{L}_{1,1}^{\mathrm{cl}} \leq \mathrm{L}_{1, \mathrm{~d}}<1.16 \mathrm{~L}_{1, \mathrm{~d}}^{\mathrm{cl}}$.

Proof of Theorem 4.1. We apply an induction argument similar to the one used in [17]. For $d=1$ and $1 / 2 \leq \gamma<3 / 2$ the bound (4.5) is identical to (3.9).

Consider the operator (2.3) in the (external) dimension $d$. We rewrite the quadratic form $h[u, u]+v[u, u]$ for $u \in H^{1}\left(\mathbb{R}^{d}, \mathbf{G}\right)$ as

$$
\begin{aligned}
h[u, u]+v[u, u] & =\int_{-\infty}^{+\infty} h\left(x_{d}\right)[u, u] d x_{d}+\int_{-\infty}^{+\infty} w\left(x_{d}\right)[u, u] d x_{d}, \\
h\left(x_{d}\right)[u, u] & =\int_{\mathbb{R}^{d-1}}\left\|\frac{\partial u}{\partial x_{d}}\right\|_{G}^{2} d x_{1} \cdots x_{d-1}, \\
w\left(x_{d}\right)[u, u] & =\int_{\mathbb{R}^{d-1}}\left[\sum_{j=1}^{d-1}\left\|\frac{\partial u}{\partial x_{j}}\right\|_{G}^{2}+\langle V(x) u, u\rangle_{G}\right] d x_{1} \cdots x_{d-1}
\end{aligned}
$$


The form $w\left(x_{d}\right)$ is closed on $\mathrm{H}^{1}\left(\mathbb{R}^{\mathrm{d}-1}, \mathbf{G}\right)$ for a.e. $\mathrm{x}_{\mathrm{d}} \in \mathbb{R}$ and it induces the self-adjoint operator

$$
W\left(x_{d}\right)=-\sum_{k=1}^{d-1} \frac{\partial^{2}}{\partial x_{k}^{2}} \otimes 1_{G}+V\left(x_{1}, \ldots, x_{d-1} ; x_{d}\right)
$$

on $\mathrm{L}^{2}\left(\mathbb{R}^{\mathrm{d}-1}, \mathbf{G}\right)$. For a fixed $x_{\mathrm{d}} \in \mathbb{R}$ this is a Schrödinger operator in $\mathrm{d}-1$ dimensions. Its negative spectrum is discrete, hence $W_{-}\left(x_{d}\right)$ is compact on $\mathrm{L}^{2}\left(\mathbb{R}^{\mathrm{d}-1}, \mathbf{G}\right)$.

Assume that we have (4.4)-(4.5) for the dimension $d-1$ and all $\gamma$ from the interval $1 / 2 \leq \gamma<3 / 2$. Then $\operatorname{tr} W_{-}^{\gamma+\frac{1}{2}}\left(x_{d}\right)$ satisfies the bound

$$
\operatorname{tr} W_{-}^{\gamma+\frac{1}{2}}\left(x_{d}\right) \leq L_{\gamma+\frac{1}{2}, d-1} \int_{\mathbb{R}^{d-1}} \operatorname{tr} V_{-}^{\gamma+\frac{d}{2}}\left(x_{1}, \ldots, x_{d-1} ; x_{d}\right) d x_{1} \cdots d x_{d-1}
$$

for a.e. $x_{d} \in \mathbb{R}$. Here

$$
\begin{array}{cll}
\mathrm{L}_{\gamma+\frac{1}{2}, \mathrm{~d}-1}=\mathrm{L}_{\gamma+\frac{1}{2}, \mathrm{~d}-1}^{\mathrm{cl}} & \text { for } & \gamma \geq 1, \\
\mathrm{~L}_{\gamma+\frac{1}{2}, \mathrm{~d}-1} \leq 2 \mathrm{~L}_{\gamma+\frac{1}{2}, \mathrm{~d}-1}^{\mathrm{cl}} & \text { for } & 1 / 2 \leq \gamma<1 .
\end{array}
$$

Indeed, (4.8) follows from (4.3) and (4.9) follows from (4.4)-(4.5) in dimension $\mathrm{d}-1$.

Let $w_{-}\left(x_{d}\right)[\cdot, \cdot]$ be the quadratic form corresponding to the operator $W_{-}\left(x_{d}\right)$ on $\mathrm{H}=\mathrm{L}^{2}\left(\mathbb{R}^{\mathrm{d}-1}, \mathbf{G}\right)$. We have $w\left(x_{d}\right)[u, u] \geq-w_{-}\left(x_{d}\right)[u, u]$ and

$$
h[u, u]+v[u, u] \geq \int_{-\infty}^{+\infty}\left[\left\|\frac{\partial u}{\partial x_{d}}\right\|_{H}^{2}-\left\langle W_{-}\left(x_{d}\right) u, u\right\rangle_{H}\right] d x_{d}
$$

for all $u \in H^{1}\left(\mathbb{R}^{d}, \mathbf{G}\right)$. According to section 2.2 the form on the r.h.s. of $(4.10)$ can be closed to $H^{1}(\mathbb{R}, \mathbf{H})$ and induces the self-adjoint operator

$$
-\frac{d^{2}}{d x_{d}^{2}} \otimes 1_{H}-W_{-}\left(x_{d}\right)
$$

on $L^{2}(\mathbb{R}, \mathbf{H})$. Then $(4.10)$ implies

$$
\operatorname{tr}\left(-\Delta \otimes 1_{G}+V\right)_{-}^{\gamma} \leq \operatorname{tr}\left(-\frac{d^{2}}{d x_{d}^{2}} \otimes 1_{H}-W_{-}\left(x_{d}\right)\right)_{-}^{\gamma} .
$$

The assumption $V \in L^{\gamma+\frac{d}{2}}\left(\mathbb{R}^{d}\right)$ implies that $\operatorname{tr} W_{-}^{\gamma+\frac{1}{2}}$ is an integrable function and we can apply Corollary 3.5 to the r.h.s. of (4.11). In view of (4.7) 
we find

$$
\begin{aligned}
\operatorname{tr}\left(-\frac{\mathrm{d}^{2}}{\mathrm{~d} x_{\mathrm{d}}^{2}} \otimes \mathbf{1}_{\mathrm{H}}-\mathrm{W}_{-}\left(\mathrm{x}_{\mathrm{d}}\right)\right)_{-}^{\gamma} & \leq \mathrm{L}_{\gamma, 1} \int_{-\infty}^{+\infty} \operatorname{tr} \mathrm{W}_{-}^{\gamma+\frac{1}{2}}\left(x_{\mathrm{d}}\right) \mathrm{d} x_{\mathrm{d}} \\
& \leq \mathrm{L}_{\gamma, 1} \mathrm{~L}_{\gamma+\frac{1}{2}, \mathrm{~d}-1} \int_{\mathbb{R}^{\mathrm{d}}} \operatorname{tr} \mathrm{V}_{-}^{\gamma+\frac{\mathrm{d}}{2}} \mathrm{~d} x
\end{aligned}
$$

for $\gamma \geq 1 / 2$. The bounds (4.5), (4.8) or (4.9) and the calculation

$$
\begin{aligned}
\mathrm{L}_{\gamma, 1}^{\mathrm{cl}} \mathrm{L}_{\gamma+\frac{1}{2}, \mathrm{~d}-1}^{\mathrm{cl}} & =\frac{\Gamma(\gamma+1)}{2 \pi^{\frac{1}{2}} \Gamma\left(\gamma+\frac{1}{2}+1\right)} \cdot \frac{\Gamma\left(\gamma+\frac{1}{2}+1\right)}{2^{\mathrm{d}-1} \pi^{\frac{\mathrm{d}-1}{2}} \Gamma\left(\gamma+\frac{1}{2}+\frac{\mathrm{d}-1}{2}+1\right)} \\
& =\frac{\Gamma(\gamma+1)}{2^{\mathrm{d}} \pi^{\frac{\mathrm{d}}{2}} \Gamma\left(\gamma+\frac{\mathrm{d}}{2}+1\right)}=\mathrm{L}_{\gamma, \mathrm{d}}^{\mathrm{cl}}
\end{aligned}
$$

complete the proof.

4.2. Estimates for magnetic Schrödinger operators. Following a remark by B. Helffer [13] and using the arguments from [17] we can extend Theorem 4.1 to Schrödinger operators with magnetic fields. Let $Q(\boldsymbol{a})$ be a self-adjoint operator in $\mathrm{L}^{2}\left(\mathbb{R}^{\mathrm{d}}, \mathbf{G}\right)$

$$
\mathrm{Q}(\boldsymbol{a})=(i \nabla+\mathbf{a}(x))^{2} \otimes \mathbf{1}_{\mathbf{G}}+\mathrm{V}(x),
$$

where

$$
a(x)=\left(a_{1}(x), \cdots, a_{d}(x)\right)^{t}, \quad d \geq 2,
$$

is a magnetic vector potential with real-valued entries $a_{k} \in L_{\text {loc }}^{2}\left(\mathbb{R}^{d}\right)$.

We consider the inequality

$$
\operatorname{tr}(\mathrm{Q}(\boldsymbol{a}))_{-}^{\gamma} \leq \tilde{\mathrm{L}}_{\gamma, \mathrm{d}} \int_{\mathbb{R}^{\mathrm{d}}} \mathrm{V}_{-}^{\frac{\mathrm{d}}{2}+\gamma} \mathrm{dx}
$$

where the nonpositive operator function $\mathrm{V}(\cdot)$ satisfies (4.1). In [17] it has been shown, that

$$
\tilde{\mathrm{L}}_{\gamma, \mathrm{d}}=\mathrm{L}_{\gamma, \mathrm{d}}^{\mathrm{cl}} \quad \text { for all } \quad \gamma \geq 3 / 2, \quad \mathrm{~d} \in \mathbb{N} .
$$

In general, the sharp constant $\tilde{\mathrm{L}}_{\gamma, \mathrm{d}}$ in (4.14) might differ from the sharp constant $\mathrm{L}_{\gamma, \mathrm{d}}$ in 4.2 )

$$
\mathrm{L}_{\gamma, \mathrm{d}}^{\mathrm{cl}} \leq \mathrm{L}_{\gamma, \mathrm{d}} \leq \tilde{\mathrm{L}}_{\gamma, \mathrm{d}} .
$$

By combining the arguments from [17] and those used in the prove of Theorem 4.1 we immediately obtain the following result: 
Theorem 4.2. The following estimates on the sharp constants $\tilde{\mathrm{L}}_{\gamma, \mathrm{d}}$ in (4.13) hold

$$
\begin{aligned}
& \tilde{\mathrm{L}}_{\gamma, \mathrm{d}} \leq 2 \mathrm{~L}_{\gamma, \mathrm{d}}^{\mathrm{cl}} \quad \text { for all } \quad 1 \leq \gamma<3 / 2, \quad \mathrm{~d} \geq 2 \text {, } \\
& \tilde{\mathrm{L}}_{\gamma, \mathrm{d}} \leq 4 \mathrm{~L}_{\gamma, \mathrm{d}}^{\mathrm{cl}} \quad \text { for all } \quad 1 / 2 \leq \gamma<1, \mathrm{~d} \geq 2 \text {. }
\end{aligned}
$$

5. TRACE FORMULAE AND ESTIMATES FROM BELOW FOR $d=1$.

5.1. Matrix-valued potentials. Let $\mathbf{G}=\mathbb{C}^{n}$ be a finite dimensional Hilbert space. We consider the system of ordinary differential equations

$$
-\left(\frac{d^{2}}{d x^{2}} \otimes 1\right) y(x)+V(x) y(x)=k^{2} y(x), \quad x \in \mathbb{R}
$$

where $\mathrm{V}$ is a compactly supported, smooth (not necessary sign definite) Hermitian matrix-valued function. Define

$$
\chi_{\min }:=\min \operatorname{supp} \mathrm{V} \text { and } x_{\max }:=\max \operatorname{supp} \mathrm{V} .
$$

Then for any $k \in \mathbb{C} \backslash\{0\}$ there exist unique $n \times n$ matrix-solutions $F(x, k)$ and $G(x, k)$ of the equations

$$
\begin{aligned}
-F_{x x}^{\prime \prime}(x, k)+V F(x, k) & =k^{2} F(x, k), \\
-G_{x x}^{\prime \prime}(x, k)+\operatorname{VG}(x, k) & =k^{2} G(x, k),
\end{aligned}
$$

satisfying

$$
\begin{array}{lll}
\mathrm{F}(\mathrm{x}, \mathrm{k})=\mathrm{e}^{i k x} \mathbf{1}_{\mathbf{G}} & \text { as } & x \geq x_{\max }, \\
\mathrm{G}(x, \mathrm{k})=\mathrm{e}^{-i k x} \mathbf{1}_{\mathrm{G}} \quad \text { as } & x \leq x_{\min } .
\end{array}
$$

If $k \in \mathbb{C} \backslash\{0\}$, then the pairs of matrices $F(x, k), F(x,-k)$ and $G(x, k)$, $\mathrm{G}(x,-\mathrm{k})$ form full systems of independent solutions of (5.1). Hence the matrix $F(x, k)$ can be expressed as a linear combination of $G(x, k)$ and $\mathrm{G}(\mathrm{x},-\mathrm{k})$

$$
F(x, k)=G(x, k) B(k)+G(x,-k) A(k) .
$$

The matrix functions $A(k)$ and $B(k)$ are uniquely defined by (5.4).

5.2. Trace formulae. In [17] the Buslaev-Faddeev-Zakharov trace formulae were generalized for the matrix-valued potentials satisfying the conditions from the previous subsection. We recall here the first three trace 
identities given by the equations (1.60)-(1.62) from [17]

$$
\begin{aligned}
\frac{1}{4} \int_{-\infty}^{+\infty} \operatorname{tr} \mathrm{V} \mathrm{d} x & =\mathrm{I}_{0}-\sum_{\mathrm{l}=1}^{\mathrm{N}} \mathrm{E}_{\mathrm{l}}^{1 / 2}, \\
\frac{3}{16} \int_{-\infty}^{+\infty} \operatorname{tr} \mathrm{V}^{2} \mathrm{~d} \mathrm{x} & =3 \mathrm{I}_{2}+\sum_{\mathrm{l}=1}^{\mathrm{N}} \mathrm{E}_{\mathrm{l}}^{3 / 2}, \\
\frac{5}{32} \int_{-\infty}^{+\infty} \operatorname{tr} \mathrm{V}^{3} \mathrm{~d} x+\frac{5}{64} \int_{-\infty}^{+\infty} \operatorname{tr}\left(\frac{\mathrm{dV}}{\mathrm{d} x}\right)^{2} \mathrm{~d} \mathrm{x} & =5 \mathrm{I}_{4}-\sum_{\mathrm{l}=1}^{\mathrm{N}} \mathrm{E}_{\mathrm{l}}^{5 / 2},
\end{aligned}
$$

where

$$
I_{j}=(2 \pi)^{-1} \int_{-\infty}^{+\infty} k^{j} \ln |\operatorname{det} A(k)| d k \quad j=0,2,4 .
$$

Note that for real k's we have (cf. (1.11) in [17])

$$
A(k) A^{*}(k)=1_{G}+B(-k) B^{*}(-k)
$$

Thus we obtain $|\operatorname{det} A(k)| \geq 1$ for all $k \in \mathbb{R}$ and

$$
I_{j} \geq 0 \quad j=0,2,4 .
$$

Remark. Notice that

$$
\mathrm{L}_{1 / 2,1}^{\mathrm{cl}}=1 / 4, \quad \mathrm{~L}_{3 / 2,1}^{\mathrm{cl}}=3 / 16, \quad \mathrm{~L}_{5 / 2,1}^{\mathrm{cl}}=5 / 32 .
$$

5.3. $\gamma=1 / 2$. The identity (5.5) immediately leads to a bound from below on the sum of the square roots of the operator (5.1). Indeed, (5.8) implies

$$
\mathrm{L}_{1 / 2,1}^{\mathrm{cl}} \int\left(\operatorname{tr} \mathrm{V}_{-}-\operatorname{tr} \mathrm{V}_{+}\right) \mathrm{d} x \leq \sum_{\mathrm{l}} \mathrm{E}_{\mathrm{l}}^{1 / 2}
$$

For the scalar case this estimate has been pointed out in [11], see also [25]. By continuity this bound extends to all matrix functions $V$, for which

$$
\operatorname{tr} \mathrm{V}_{+}(\cdot) \in \mathrm{L}^{1}(\mathbb{R}) \quad \text { and } \quad \operatorname{tr} \mathrm{V}_{-}(\cdot) \in \mathrm{L}^{1}(\mathbb{R}) .
$$

Using a standard density argument and (3.1) we conclude, that (5.10) holds also for general separable Hilbert spaces $\mathbf{G}$. This implies

Corollary 5.1. Let $\mathrm{V}(\mathrm{x}) \in \mathrm{S}_{1}(\mathbf{G})$ and $\operatorname{tr} \mathrm{V}_{ \pm}(\cdot) \in \mathrm{L}_{1}(\mathbb{R})$. Then for the $1 / 2$ moments of the negative eigenvalues of the operator (2.3) we have the following two side inequalities

$$
\mathrm{L}_{1 / 2,1}^{\mathrm{cl}} \int\left(\operatorname{tr} \mathrm{V}_{-}-\operatorname{tr} \mathrm{V}_{+}\right) \mathrm{d} \mathrm{x} \leq \sum_{\mathrm{l}} \mathrm{E}_{\mathrm{l}}^{1 / 2} \leq 2 \mathrm{~L}_{1 / 2,1}^{\mathrm{cl}} \int \operatorname{tr} \mathrm{V}_{-} \mathrm{d} x
$$


5.4. $\gamma=3 / 2$. Let us return to the case $\mathbf{G}=\mathbb{C}^{n}$ and let $V$ be a smooth, compactly supported matrix-valued function. The upper bound (3.1) and the identity (5.5) imply

$$
\begin{aligned}
\mathrm{I}_{1} & =\sum_{\mathrm{l}=1}^{\mathrm{N}} \mathrm{E}_{\mathrm{l}}^{1 / 2}+\mathrm{L}_{1 / 2,1}^{\mathrm{cl}} \int\left(\operatorname{tr} \mathrm{V}_{+}-\operatorname{tr} \mathrm{V}_{-}\right) \mathrm{dx} \\
& \leq \mathrm{L}_{1 / 2,1}^{\mathrm{cl}} \int\left(\operatorname{tr} \mathrm{V}_{+}+\operatorname{tr} \mathrm{V}_{-}\right) \mathrm{d} x .
\end{aligned}
$$

Moreover, from (4.3) with $\mathrm{d}=1$ and $\gamma=5 / 2$, (5.7) and (5.9) it follows that

$$
\begin{aligned}
5 \mathrm{I}_{4} & =\mathrm{L}_{5 / 2,1}^{\mathrm{cl}} \int\left(\operatorname{tr} \mathrm{V}_{+}^{3}-\operatorname{tr} \mathrm{V}_{-}^{3}\right) \mathrm{d} x+\frac{1}{2} \mathrm{~L}_{5 / 2,1}^{\mathrm{cl}} \int \operatorname{tr}\left(\frac{\mathrm{dV}}{\mathrm{d} x}\right)^{2} \mathrm{~d} x+\sum_{\mathrm{l}} \mathrm{E}_{\mathrm{l}}^{5 / 2} \\
& \leq \mathrm{L}_{5 / 2,1}^{\mathrm{cl}} \int \operatorname{tr} \mathrm{V}_{+}^{3} \mathrm{~d} x+\frac{1}{2} \mathrm{~L}_{5 / 2,1}^{\mathrm{cl}} \int \operatorname{tr}\left(\frac{\mathrm{dV}}{\mathrm{d} x}\right)^{2} \mathrm{~d} x .
\end{aligned}
$$

Note that in the scalar case the inequalities (5.12) and (5.13) with somewhat worse constant were found in [25] and [21] respectively. These estimates together with Hölder's inequality give

$\mathrm{I}_{2} \leq \mathrm{I}_{0}^{1 / 2} \mathrm{I}_{4}^{1 / 2}=\frac{1}{16}\left[\int\left(\operatorname{tr} \mathrm{V}_{+}+\operatorname{tr} \mathrm{V}_{-}\right) \mathrm{dx}\right]^{\frac{1}{2}}\left[2 \int \operatorname{tr} \mathrm{V}_{+}^{3} \mathrm{dx}+\int \operatorname{tr}\left(\frac{\mathrm{dV}}{\mathrm{d} x}\right)^{2} \mathrm{dx}\right]^{\frac{1}{2}}$

Inserting (5.14) into (5.6) and considering the special case $\mathrm{V}_{+}=0$, we find

$$
\frac{3}{16} \int \operatorname{tr} V_{-}^{2} d x-\sum_{l} E_{l}^{3 / 2} \leq \frac{3}{16}\left[\int \operatorname{tr} V_{-} d x\right]^{\frac{1}{2}}\left[\int \operatorname{tr}\left(\frac{d V}{d x}\right)^{2} d x\right]^{\frac{1}{2}} .
$$

Standard density and continuity arguments allow us to extend (5.15) to general separable Hilbert spaces $\mathbf{G}$ and arbitrary nonpositive operator-valued potentials $\mathrm{V}$, for which all integrals in (5.15) are finite.

5.5. A remainder term. Let us discuss further the inequality (5.15). First note, that in view of (4.3) for $d=1$ and $\gamma=3 / 2$, the 1.h.s. of (5.15) is nonnegative. Therefore the inequalities $(5.15)$ can be interpreted as an estimate on the difference between the sum $\sum_{l} E_{l}^{3 / 2}$ and the classical phase space integral

$$
\mathrm{L}_{3 / 2}^{\mathrm{cl}} \int \operatorname{tr} \mathrm{V}_{-}^{2} \mathrm{~d} x=\iint \operatorname{tr}\left(\mathrm{p}^{2}+\mathrm{V}(\mathrm{x})\right)_{-}^{3 / 2} \frac{\mathrm{dpd} x}{2 \pi}
$$

By replacing $\mathrm{V}$ by $\alpha \mathrm{V}$ we obtain the following result: 
Theorem 5.2. Assume that $\mathrm{V}$ is a nonpositive operator-valued function such that $\operatorname{tr} \mathrm{V}_{-} \in \mathrm{L}^{1}(\mathbb{R}) \cap \mathrm{L}^{2}(\mathbb{R})$ and $\operatorname{tr}(\mathrm{dV} / \mathrm{dx})^{2} \in \mathrm{L}^{1}(\mathbb{R})$. Then

$$
\operatorname{tr}\left(-\frac{\mathrm{d}^{2}}{\mathrm{~d} x^{2}} \otimes 1_{\mathrm{G}}+\alpha \mathrm{V}\right)_{-}^{3 / 2}=\alpha^{2} \mathrm{~L}_{3 / 2,1}^{\mathrm{cl}} \int \operatorname{tr} \mathrm{V}_{-}^{2} \mathrm{~d} x-\mathrm{R}(\alpha)
$$

for all $\alpha>0$, where

$$
0 \leq R(\alpha) \leq \frac{3 \alpha^{3 / 2}}{16}\left[\int \operatorname{tr} V_{-} d x\right]^{\frac{1}{2}}\left[\int \operatorname{tr}\left(\frac{d V}{d x}\right)^{2} d x\right]^{\frac{1}{2}}
$$

Remark. For large values of the coupling constant $\alpha$, Theorem 5.2 gives us the correct order $\mathrm{O}\left(\alpha^{3 / 2}\right)$ of the remainder term in the Weyl asymptotic formula for 3/2-moments of the negative eigenvalues.

5.6. Acknowledgements. The second and the fourth authors wish to express their gratitude to B.Helffer for his valuable comments on magnetic Schrödinger operators. D.Hundertmark thanks the Mathematical Department of the Royal Institute of Technology in Stockholm for its warm hospitality and the Deutsche Forschungsgemeinschaft for financial support under grant $\mathrm{Hu}$ 773/1-1. A.Laptev has been supported by the Swedish Natural Sciences Research Council, Grant M-AA/MA 09364-320, T.Weidl has been supported by the Swedish Natural Science Council dnr 11017-303.

Partial financial support from the European Union through the TMR network FMRX-CT 96-0001 is gratefully acknowledged.

\section{REFERENCES}

[1] Aizenman M. and Lieb E.H.: On semi-classical bounds for eigenvalues of Schrödinger operators. Phys. Lett. 66A, 427-429 (1978)

[2] Ando T: Majorization, doubly stochastic matrices, and comparison of eigenvalues. Linear Algebra Appl. 118 , 163-248 (1989)

[3] Bhatia R.: Matrix analysis. Springer Graduate Texts in Mathematics, 169. Springer 1997

[4] Birman M.S.: The spectrum of singular boundary problems. (Russian) Mat. Sb. (N.S.) 55 (97), 125-174 (1961). (English) Amer. Math. Soc. Transl. 53, 23-80 (1966)

[5] Blanchard Ph. and Stubbe J.: Bound states for Schrödinger Hamiltonians: Phase Space Methods and Applications. Rev. Math. Phys., 35, 504-547 (1996)

[6] Buslaev V.S. and Faddeev L.D: Formulas for traces for a singular Sturm-Liouville differential operator. [English translation], Dokl. AN SSSR, 132, 451-454 (1960)

[7] Conlon J.G.: A new proof of the Cwikel-Lieb-Rosenbljum bound. Rocky Mountain J. Math., 15, 117-122 (1985)

[8] Cwikel M.: Weak type estimates for singular values and the number of bound states of Schrödinger operators. Trans. AMS, 224, 93-100 (1977)

[9] Faddeev L.D. and Zakharov V.E.: Korteweg-de Vries equation: A completely integrable hamiltonian system. Func. Anal. Appl., 5, 18-27 (1971)

[10] Fan K.: Maximum properties and inequalities for the eigenvalues of completely continuous operators. Proc. Nat. Acad. Sci. , 37, 760-766 (1951) 
[11] Glaser V., Grosse H. and Martin A.: Bounds on the number of eigenvalues of the Schrödinger operator. Commun. Math. Phys., 59, 197-212 (1978)

[12] Gohberg I.C. and Krein M.G:: Introduction to the theory of linear non-self-adjoint operators. Trans. Math. Monographs vol 18. AMS 1969

[13] Helffer B.: private communication

[14] Helffer B. and Robert D.: Riesz means of bounded states and semi-classical limit connected with a Lieb-Thirring conjecture I,II. I -Jour. Asymp. Anal., 3, 91-103 (1990), II - Ann. de l'Inst. H. Poincare, 53 (2), 139-147 (1990)

[15] Hundertmark D., Lieb E.H. and Thomas L.E.: A sharp bound for an eigenvalue moment of the one-dimensional Schrödinger operator. Adv. Theor. Math. Phys. 2, 719731 (1998)

[16] Laptev A.: Dirichlet and Neumann Eigenvalue Problems on Domains in Euclidean Spaces. J. Func. Anal., 151, 531-545 (1997)

[17] Laptev A., Weidl T.: Sharp Lieb-Thirring inequalities in high dimensions. accepted by Acta Mathematica

[18] Li P. and Yau S.-T.: On the Schrödinger equation and the eigenvalue problem. Comm. Math. Phys., 88, 309-318 (1983)

[19] Lieb, E.H.: The number of bound states of one body Schrödinger operators and the Weyl problem. Bull. Amer. Math. Soc., 82, 751-753 (1976)

[20] Lieb, E.H.: On characteristic exponents in turbulence. Comm. Math. Phys., 82, 473480 (1984)

[21] Lieb E.H. and Thirring, W.: Inequalities for the moments of the eigenvalues of the Schrödinger Hamiltonian and their relation to Sobolev inequalities. Studies in Math. Phys., Essays in Honor of Valentine Bargmann., Princeton, 269-303 (1976)

[22] Rozenblum, G.V.: Distribution of the discrete spectrum of singular differential operators. Dokl. AN SSSR, 202, 1012-1015 (1972), Izv. VUZov, Matematika, 1, 75-86 (1976)

[23] Ruelle D.: Large volume limit of the distribution of characteristic exponents in turbulence. Comm. Math. Phys., 87, 287-302 (1982)

[24] Y.Schwinger: On the bound states for a given potential. Proc. Nat. Acad. Sci. U.S.A. 47 (1961), 122-129.

[25] Weidl, T.: On the Lieb-Thirring constants $\mathrm{L}_{\gamma, 1}$ for $\gamma \geq 1 / 2$. Comm. Math. Phys., 178, 135-146 (1996)

\begin{tabular}{|c|c|}
\hline Departments of Physics, Jadwin Hall ${ }^{1}$ & Royal Institute of Technology ${ }^{2}$ \\
\hline Princeton Universit & Department of Mathematics \\
\hline rinceton, New Jersey 08544, U.S.A & S-10044 Stockholm, Sweden \\
\hline
\end{tabular}

Universität Regensburg ${ }^{3}$

Naturwissenschaftliche Fakultät I

D-93040 Regensburg, Germany

E-mail address: hdirk@princeton.edu, laptev@math.kth.se, weidl@math.kth.se 